\title{
The role of streamers in the deflection of coronal mass ejections
}

\author{
F. P. Zuccarello ${ }^{1,2,3}$, A. Bemporad ${ }^{4}$, C. Jacobs ${ }^{1}$, M. Mierla ${ }^{5,6}$, \\ S. Poedts ${ }^{1}$, and F. Zuccarello ${ }^{3}$ \\ ${ }^{1}$ Centre for Plasma Astrophysics, K.U. Leuven, Belgium. \\ ${ }^{2}$ INAF - Osservatorio Astrofisico di Catania, via S. Sofia 78, 95123 Catania, Italy. \\ ${ }^{3}$ Dipartimento di Fisica e Astronomia, Sezione Astrofisica, Via S. Sofia 78, 95123 Catania, \\ Italy. \\ ${ }^{4}$ Istituto Nazionale di Astrofisica (INAF), Osservatorio Astronomico di Torino, Strada \\ Osservatorio 20, 10025 Pino Torinese, Torino, Italy. \\ ${ }^{5}$ Institute of Geodynamics of the Romanian Academy, Bucharest, Romania. \\ ${ }^{6}$ Royal Observatory of Belgium, Brussels, Belgium.
}

\begin{abstract}
On 2009 September 21, a filament eruption and the associated Coronal Mass Ejection (CME) was observed by the STEREO spacecraft. The CME originated from the southern hemisphere and showed a deflection of about $15^{\circ}$ towards the heliospheric current sheet (HCS) during its propagation in the COR1 field-of-view (FOV). The aim of this paper is to provide a physical explanation for the strong deflection of the CME. We first use the STEREO observations in order to reconstruct the three dimensional (3D) trajectory of the CME. Starting from a magnetic configuration that closely resembles the potential field extrapolation for that date, we performed numerical magneto-hydrodynamics (MHD) simulations. By applying localized shearing motions, a CME is initiated in the simulation, showing a similar non-radial evolution, structure, and velocity as the observed event. The CME gets deflected towards the current sheet of the larger northern helmet streamer, due to an imbalance in the magnetic pressure and tension forces and finally it gets into the streamer and propagates along the heliospheric current sheet.
\end{abstract}

Keywords. Sun: coronal mass ejections (CMEs), methods: numerical, Sun: corona, Sun: magnetic fields

\section{Introduction}

Since the Skylab and Solar Maximum Mission (SMM) era (e.g. MacQueen, Hundhausen \& Conover 1986), the occurrence of latitudinal deflections of coronal mass ejections (CMEs) towards the equator is a well known phenomenon, as well as similar deflections of flare associated shock waves (e.g. Fengsi \& Dryer 1991). Later on, in the SOlar and Heliospheric Observatory (SOHO) era, many detailed investigations of deflections have been performed: statistical results show that during solar minima, CME deflections occur preferentially towards the equator, while during periods of intense solar activity both deflection towards the equator and towards the poles are observed, depending on the location and total area of coronal holes (Cremades, Bothmer \& Tripathi 2006). Recently, Lopez et al. (2011, IAU Symposium 286, poster contribution) investigated the deflection of CMEs during the two previous solar minima. The authors found that between $60-75 \%$ of the studied events exibit a deflection towards the nearest streamer independently of which solar minimum is considered. This indicates that the same physical mechanism could be responsable for the observed deflection of CMEs. 

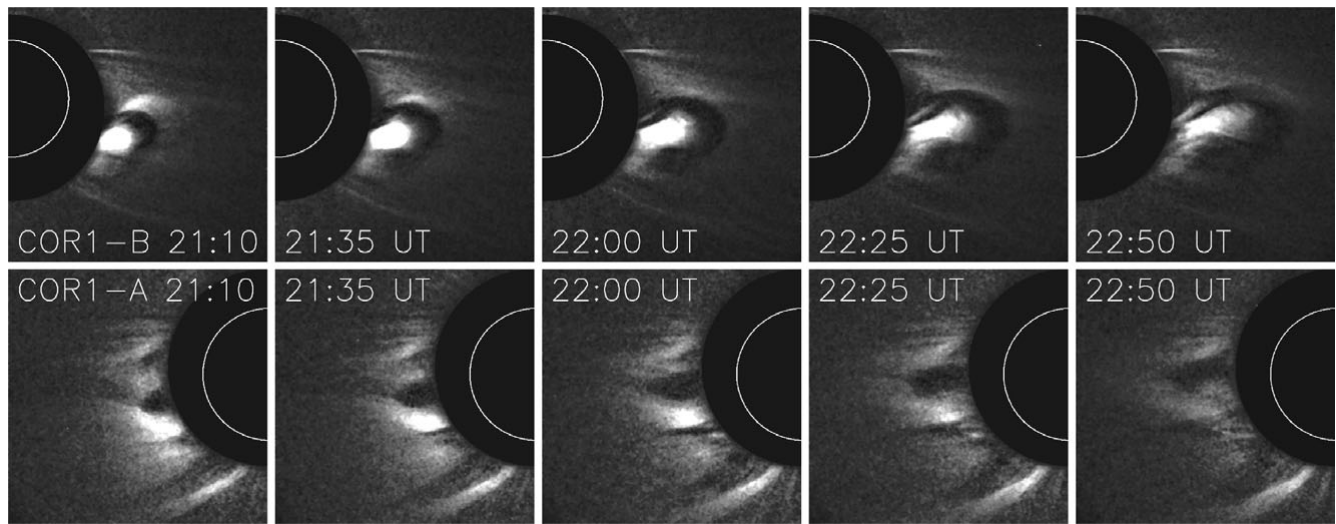

Figure 1. Sequence of COR1 images acquired during the CME propagation by STEREO-B (top) and -A (bottom). The COR1-B images show a three-part CME composed of a bright leading edge, the dark cavity and the bright core. The three-part components are not visible in the corresponding COR1-A images (bottom panels), where a more diffuse structure is observed. This figure is published in Zuccarello et al. (2012).

The aim of this paper is to provide a physical explanation for the deflection of the CME observed on 2009 September 21. In order to do this, we use Solar TErrestrial RElations Observatory (STEREO; see Kaiser et al. 2008) observations to reconstruct the three dimensional dynamics of the CME and combine these with MHD simulations of this event.

\section{Observations}

On 2009 September 21, a small prominence eruption leading to a CME occurred. This eruption has been observed by both the Extreme UV Imagers (EUVI) and the COR1 coronagraphs aboard the twin STEREO spacecraft.

The CME was better observed in COR1-B images as a classical three-part structure event, with a bright leading-edge, a dark cavity and a bright core (Fig. 1, top panels). The CME entered in the instrument field-of-view (FOV) around 19:45 UT being observed (as a three-part structure) until 00:10 UT on September 22, while the erupting core was visible until $\sim$ 01:35 UT. The CME core first appeared above the COR1 occulter at a projected latitude of $\simeq 25^{\circ}$ South. The core expanded northward until $\sim 22: 30 \mathrm{UT}$, when the top of the core was at a projected latitude of $15^{\circ}$ South, i.e. closer to the equatorial plane. Interestingly, between 22:30 UT and 23:00 UT the CME core underwent a further and faster migration toward the equator, eventually approaching it. The CME was finally observed on 2009 September 22 by the COR2-B instrument as a faint threepart structured bubble expanding along the equatorial plane.

The CME was much more diffuse in COR1-A images and the three-part components were not clearly observed as compared with COR1-B images (Fig. 1, bottom panels). This is likely due to the large separation angle between the STEREO-A and -B spacecraft, making the CME, which expanded closer to the STEREO-B plane of the sky, very faint in the STEREO-A data.

\section{Simulations}

Using the two vantage points of the STEREO spacecraft, we reconstructed the 3D trajectory of the CME (see Zuccarello et al. (2012) for more details). We found that 


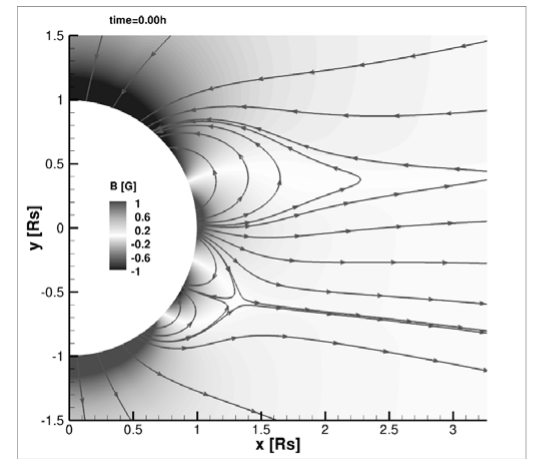

(a)

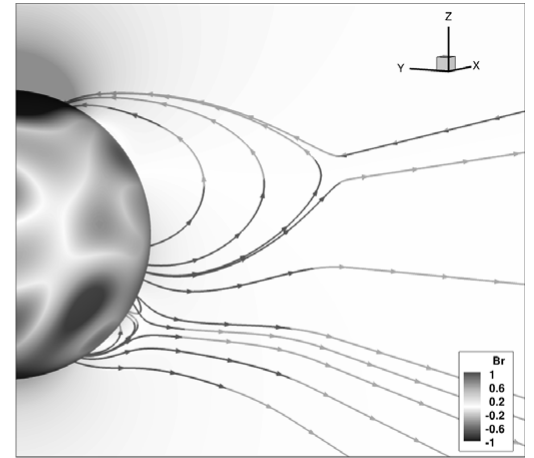

(b)

Figure 2. Magnetic field distribution (colour scale) and selected field lines for (a) the steady state of the simulation and (b) the PFSS extrapolation obtained from the MDI data on 2009 September 19 at 12:04 UT. This figure is published in Zuccarello et al. (2012). A color version of the figure is available in the online version.

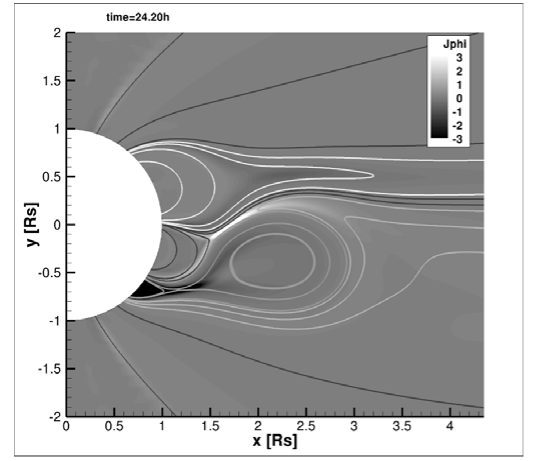

(a)

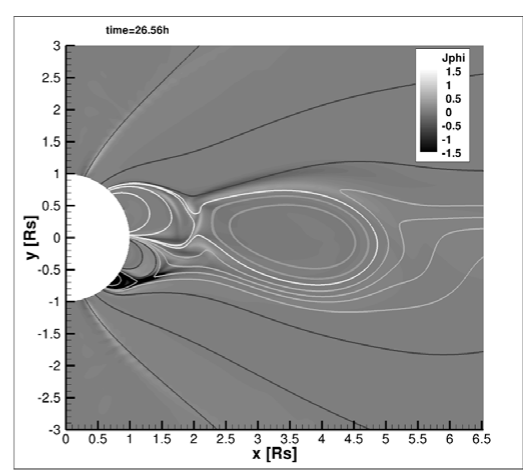

(b)

Figure 3. Snapshots of the time evolution of the azimuthal component of the current density (gray color scale) overplotted with some selected field lines. Different flux systems are identified by different colors (see text for more details). This figure is published in Zuccarello et al. (2012). A color version of the figure is available in the online version.

during its propagation the CME undergoes a longitudinal deflection not larger than $10^{\circ}$, mainly travelling along a meridional plane. Therefore, the ideal MHD equations are solved numerically on a spherical, axisymmetric $(2.5 \mathrm{D})$ domain covering the region between the solar north and south pole, i.e. $(r, \vartheta) \in\left[1 R_{\odot}, 30 R_{\odot}\right] \times[0, \pi]$.

Figure 2 shows the stationary solution for the MHD simulation (a) and the potential field source surface (PFSS) extrapolation for the 2009 September 19 (b). The initial magnetic configuration of the simulation presents a morphology similar to the reconstructed potential magnetic field. We would like to note that the key properties of the reconstructed field, i.e. the asymmetry between the two outer arcades, the northward shift of the cusp of the helmet streamer and the southern pseudostreamer, are all reproduced.

In order to form the prominence and drive the eruption, we apply localized shearing motions along the polarity inversion line of the southern loop system (Zuccarello et al. 2012). Figure 3(a) shows the magnetic configuration of the system after $21.84 \mathrm{hr}$. The grey scale denotes the azimuthal component of the current density, while the different colours of the field lines indicate different flux systems. Regions of high current density indicate the reconnection location. As a consequence of the applied shearing motions, the 


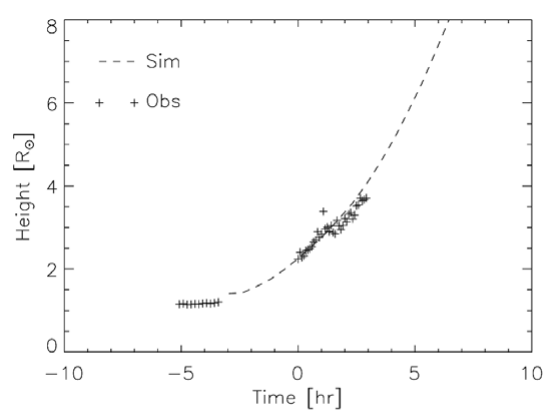

(a)

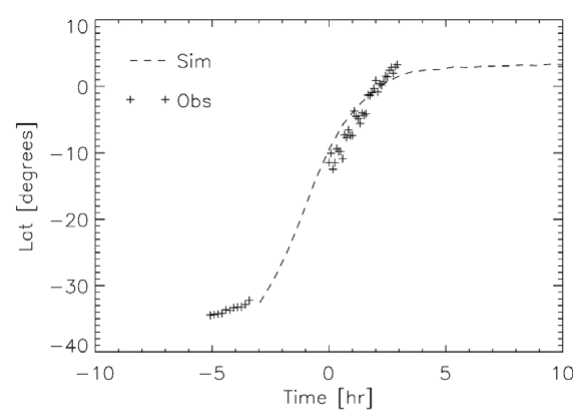

(b)

Figure 4. Comparison between the simulation (dashed line) and the observation (plus signs). (a) Altitude versus time and (b) latitude versus time. Time zero is 20:00 UT on 2009 September 21 , corresponding to the time at which the CME was at $2.25 R_{\odot}$. This figure is published in Zuccarello et al. (2012).

magnetic pressure increases and the southern side arcade starts to expand. During this expansion, the null point in the pseudostreamer is pushed northwards and one elongated current sheet is formed between the expanding southern arcade and the open field region at the north side of it, eventually initiating the magnetic reconnection. As a consequence of this reconnection, the magnetic flux of the expanding southern arcade (orange field lines) is transferred partially to the central arcade (red field lines), that becomes bigger, and partially to the open flux of the northern helmet streamer (blue field lines). The result of this process of interchange reconnection is visualized in the figure by the cyan field lines, i.e. originally closed field lines belonging to the southern arcade and that now belong to the southern coronal hole.

The pinching at the flanks of the southern arcade resulted in the formation of the flux rope (pink field lines) and during this reconnection process more and more magnetic flux is transferred from the southern arcade to the flux rope. The reconnection at the upper part of the expanding arcade results in a magnetic pressure imbalance between the north and the south part of the side arcade that, as a consequence, is deflected towards the equator. At a certain moment, due to the ongoing reconnection inside the southern arcade and the continuous growth of the central arcade, the newly formed open flux of the southern coronal hole (cyan field lines) will reconnect with the flux of the central arcade definitely separating the flux rope from its formation location and further contributing to the deflection of the CME toward the heliospheric current sheet (see Fig. 3(b)).

\section{Discussion}

In order to compare the early stages of the dynamics of the event, Fig. 4(a) shows the height-time plot for both the simulation (dashed line) and the reconstructed trajectory of the CME (plus signs). For the purpose of comparison with the observations, we set the origin of the time axis at the moment at which the core of the CME has an altitude of $2.25 R_{\odot}$ in both the simulation and the observation. The simulated flux rope has a height-time evolution that is comparable with the altitude reconstruction of the CME. For both the simulated and the observed CMEs it takes about 6 hr to reach an altitude of $4 R_{\odot}$ and both CMEs are slow.

In order to further compare the dynamics of the simulated and observed CME, in Fig. 4(b) we show the latitude-time plot for both the simulation (dashed line) and the reconstructed CME (plus signs). The prominence has a latitude of about $35^{\circ}$ south and 
at time $-5 \mathrm{hr}$ (15:00 UT on 2009 September 21) it is evolving in the EUVI-B FOV. In about one and half hour it reaches a latitude of about $31^{\circ}$ south and disappears from the EUVI FOV. The simulated flux rope starts from a location of about $33^{\circ}$ south and experiences a deflection of about $20^{\circ}$ in $3 \mathrm{hr}$, approaching an altitude of $2.25 R_{\odot}$. At this altitude the core of the observed CME is visible in the COR1 FOV and its latitudinal deflection can be followed for another three hours. At $2.25 R_{\odot}$ the core of the observed CME has a latitude of about $12^{\circ}$ south and quickly approaches a latitude of about $4^{\circ}$ north. This latitudinal behavior is well reproduced by the simulation.

Concluding, this study shows that during solar minima, as a consequence of the global magnetic field structure, even CMEs originating from high latitude can be easily deflected towards the heliospheric current sheet, eventually resulting in geoeffective events.

\section{Acknowledgements}

This research was funded by projects GOA-2009-009 (K.U.Leuven), G.0304.07 (FWO Vlaanderen), 3E090665 (FWO Vlaanderen), and C 90347 (ESA Prodex 9). The work of MM was supported from the contract TE - 73/11.08.2010. For the simulations we used the infrastructure of the VSC - Flemish Supercomputer Center, funded by the Hercules foundation and the Flemish Government - department EWI. STEREO is a NASA project. $\mathrm{SOHO}$ is a joint ESA and NASA project.

\section{References}

Cremades, H., Bothmer, V., \& Tripathi, D. 2006, Adv. Space Res., 38, 461.

Fengsi, Wei \& Dryer, Murray 1991, Solar Physics, Volume 132, Issue 2, pp. 373-394

Kaiser, M. L. et al. 2008, Space Sci. Rev., 136, 5

Lopez, F. M., Cremades, H., \& Balmaceda, L. 2003, in: C. H. Mandrini \& D. Webb (eds.), Comparative Magnetic Minima: characterizing quiet times in the Sun and stars, Proc. IAU Symposium No. 286 (Mendoza: ASP), in press.

MacQueen, R. M., Hundhausen, A. J., \& Conover, C. W. 1986, J. Geophys. Res., 91, 31

Zuccarello, F. P., Bemporad, A., Jacobs, C., Mierla, M., Poedts, S., \& Zuccarello, F. 2012, ApJ, $744,66$.

\section{Discussion}

JAnEt Luhmann: There were two STEREO viewpoints. Why did you just pick one viewpoint, instead of both viewpoints?

Francesco Zuccarello: Due to the axial symmetry, our simulations are appropriate to describe events that are seen almost on the plane-of-sky (POS) for the coronagraph. This event was seen on the limb from STEREO B; this is the reason why we selected the $\mathrm{B}$ viewpoint. However, the simulation can reproduce the reconstructed $3 \mathrm{D}$ height and latitudinal time evolution of the CME. 